\title{
Using the Web for Science in the Classroom: Online Citizen Science Participation in Teaching and Learning
}

\author{
Cathal Doyle \\ School of Information \\ Management \\ Victoria University of Wellington \\ Wellington, New Zealand \\ cathal.doyle@vuw.ac.nz \\ Markus Luczak-Roesch \\ School of Information \\ Management \\ Victoria University of Wellington \\ Wellington, New Zealand \\ markus.luczak-roesch@vuw.ac.nz
}

\author{
Rodreck David \\ School of Information \\ Management \\ Victoria University of Wellington \\ Wellington, New Zealand \\ rodreck.david@vuw.ac.nz \\ Dayle Anderson \\ School of Education \\ Victoria University of Wellington \\ Wellington, New Zealand \\ dayle.anderson@vuw.ac.nz
}

\author{
Yevgeniya Li \\ School of Information \\ Management \\ Victoria University of Wellington \\ Wellington, New Zealand \\ jane.li@vuw.ac.nz \\ Cameron M. Pierson \\ School of Information \\ Management \\ Victoria University of Wellington \\ Wellington, New Zealand \\ cameron.pierson@vuw.ac.nz
}

\begin{abstract}
Introduction: Citizen involvement in scientific projects has become a way of encouraging curiosity and greater understanding of science whilst providing an unprecedented engagement between professional scientists and the general public. In this paper we specifically focus on the impact of online citizen science (OCS) participation in the science education of primary school age children in New Zealand.

Methods: We use four exploratory cases within a broader research project to examine the nature and impact of embedding OCS projects that use web based online crowdsourcing and collaboration tools within classroom environments of primary school science learners.

Results \& Discussion: Our findings provide insights into primary school teachers' perception of OCS. They offer initial insights into how teachers embed OCS in a classroom environment, and why this improves science learning aptitudes, inquisitiveness and capabilities in primary school age children. We also notice that successfully embedding OCS projects in education is affected by the project context, how the results are disseminated, and inclusivity in socio-cultural aspects.
\end{abstract}

\section{KEYWORDS}

citizen science, online citizen science, education, learning

\section{INTRODUCTION}

Participatory science, crowd-sourced science, or citizen science (CS), are a few of the many terms that refer to the modern phenomenon of scientific endeavor which involves amateur volunteers as contributors to real-world science projects. CS projects involve citizens - who are called citizen scientists - to collect field data (e.g. take pictures of sightings of specific plants or animals), while others ask citizen scientists to classify precollected data or annotate whether some particular features can be observed (e.g. a particular pattern in a plotted graph). When these contributions are enabled by the Internet, researchers also use the term online citizen science (OCS), which is the focus for this paper.

Considerable research in this area investigates the design of successful OCS projects from the perspective of professional scientists tapping into citizen science as part of their projects, or from the perspective of facilitators of citizen science such as the providers of OCS digital platforms for crowdsourcing and collaboration [1-5]. For scientists, the questions addressed often relate to how one can ensure sustained motivation and engagement of citizens so that a project reaches its target [6]. However, this point of view is limited, only regarding OCS projects as a part of the research life-cycle of professional scientists' line of inquiry. For OCS platform providers, amassing a large number of participants and adoption is an obvious incentive to their platform business model [3]. This also means they have a vested interest that does not necessarily account for the interests of the participants themselves. This raises interesting questions such as: How do users (other than professional scientists and facilitators) who engage with OCS perceive its utility and usefulness? Do these groups have a different understanding of the purpose of OCS? Do professional scientists consider these groups when developing their OCS projects?

We consider these questions, from the perspective of primary school teachers, focusing on the impact of OCS participation on the science education of children. This is an important foundation that has a potential to provide professional scientists who design and implement OCS projects with an understanding of what is 
needed if they wish to embed their OCS projects in learning environments, whilst simultaneously benefitting teaching and learning for educators and learners. From this, we then introduce four case studies that consist of primary school teachers who embedded an OCS project into a teaching unit, where we gain a perspective of OCS from primary school teachers.

Our findings reveal that OCS is seen as an opportunity for students to engage with topics that otherwise are inaccessible in a classroom or lab setting (e.g. space), but also there is a mismatch between how OCS projects frame their suitability for in-class use (if they do that at all) and intended use as outlined by teachers. Further, teachers indicated the importance of seeing the results of contributions made and the final outcome of the project, while professional scientists are often more concerned with presenting their results to the scientific community through publication of academic papers. We also find that projects with a link to the local context are preferred by teachers and highlight an important but understudied aspect, namely the cultural responsiveness of online citizen science.

The remainder of this paper is structured as follows: We provide a brief background to the study and the broader project from which it is embedded. We review related work, discussing the conceptualisations of citizen science in offline and online environments as well as its fit in the education sector. We then explain the research methods, particularly the design of the cases used in our inquiry. Thereafter, we present our findings, with a discussion that synthesizes and links them to the current conversations about OCS in related literature. We conclude the paper by summarizing the contributions and some limitations.

\subsection{Brief Background to the Study}

Authors of this paper are involved in a larger project titled "Citizen Scientists in the Classroom: Investigating the Role of Online Citizen Science in the Science Education of School Age Children”, funded by the Teaching and Learning Research Initiative (TLRI) ${ }^{1}$ of New Zealand. This project is designed as a co-constructive partnership between researchers and four primary school teachers who are advocates of science education in New Zealand. Partnership agreements with the teachers' four schools, including necessary ethics procedures, have been obtained for this research. The four teachers have been purposefully invited to be part of this research project because of their sound understanding of the role of Science Capabilities for Citizenship ${ }^{2}$ in the New Zealand curriculum for science, and knowledge of how to implement these capabilities in primary school science classrooms. All four teachers are from the Science Teaching Leadership Programme (STLP) in New Zealand ${ }^{3}$. Their participation in the STLP means they have experience in the science learning area and an interest in promoting science among other teachers and schools. Exploratory in-depth case studies were conducted with each of the four teachers.

\footnotetext{
${ }^{1}$ http://www.tlri.org.nz/

${ }^{2}$ https://scienceonline.tki.org.nz/Science-capabilities-for-citizenship/
}

\section{REVIEW OF RELATED LITERATURE}

\subsection{Science Education}

Science education is a crucial and strategic focus of education sectors in many countries. Increasing science literacy, aptitudes and capabilities is seen as a way of both enlightening the general citizenry and developing keen interests in professional and technical skills required to drive innovativeness, scientific discovery and national development [7]. In 2014, the New Zealand government launched a national strategic initiative to improve public engagement with science and technology, and build greater scientific literacy amongst its citizenry in a project titled: A Nation of Curious Minds - He Whenua Hihiri i te Mahara [7]. The New Zealand Curriculum (NZC) introduced in 2007, already had at the core of its science learning area an aim to build students' ability to engage with science and scientific issues of interest to them, through its Participating and Contributing sub-strand [8]. The stated purpose of the science learning area as a whole is that "students explore how both the natural physical world and science itself work so that they can participate as critical, informed, and responsible citizens in a society in which science plays a significant role" [8, p.17]. This explicit embedding of science and inquiry-based learning in the NZC is just one example of how science education in general has become an integral part of many primary and secondary school curricula around the world $[9,10]$.

Given this increased importance of science education in school curricula, it is noteworthy that student engagement with science is declining, reflecting international trends [11-14]. One way of improving scientific engagement, and at the same time developing capabilities with science useful as members of society, may be through the inclusion of citizen science in school programmes which provides new opportunities for the general public to participate in real scientific projects.

\subsection{Citizen Science (CS)}

Citizen science is a scientific practice $[15,16]$ originating in the 1700s [15], involving members of the public [17-20] actively engaging with professional scientists $[16,18,20]$ in scientific work [18]. The members of the public participating can be referred to as citizen scientists $[15,16]$ or volunteers $[15,17,18,20]$. Such engagement requires following an established protocol [18] that is created by the professional scientist [21] and can include one or more of the following tasks: data collection $[15,17,20]$; data processing [22, 23]; data analysis \& interpretation [15, 17, 18]; and/or dissemination of results $[17,21]$. Outcomes from citizen science projects include advancements in scientific research [16, 19], as well as increasing the public's understanding of science $[15,19,20]$.

Based on the above understanding of citizen science, we adopt the following working definition: Citizen science is a process that involves professional scientists and citizen scientists engaging on a scientific project. This engagement follows an established protocol, created by the professional scientists, which can include

${ }^{3}$ https://royalsociety.org.nz/what-we-do/funds-and-opportunities/science-teachingleadership-programme/ 
one or more of the following tasks: data collection, data processing, data analysis \& interpretation, and/or dissemination of results. Outcomes should be advancements in scientific research, as well as an increase in the public's understanding of science.

While this provides us with an understanding, and definition of CS, online citizen science (OCS) differs in various ways. We suggest that it is important to highlight these differences, in particular in light of increasingly digital learning environments. Emphasising that digital technologies are a utility for some scientific endeavours - but not all of them (or not all stages of all of them) - bares the potential to create awareness to be critical when utilising almost ubiquitous technologies and ask whether the technologies are utilised purposefully. Thus, an overview of OCS is presented next, along with a working definition.

\subsection{Online Citizen Science (OCS)}

Since the introduction of the Internet, and advancements in technology, citizen science has evolved to move the process online [15], allowing professional scientists to engage with citizen scientists in new ways $[15,16,20]$. This includes being able to provide easier access to large datasets [15, 19]; making tools available to support engagement from citizen scientists that are geographically distributed [18-20]; enabling communication between citizen scientists [15]; and providing a wider reach to a broader audience of citizen scientists [18, 20]. These citizen science projects can be aided by technology [18] or can be completely mediated online through technology [17, 18]. This extension of citizen science has been called online citizen science $[15,17,24]$, and digital citizen science [20].

Expanding on our working definition of citizen science we adopt the following working definition of online citizen science: Online citizen science is an extension of citizen science, where the tasks to be completed are aided, or completely mediated, through the Internet. Engagement can occur in different ways such as submitting data collected by citizen scientists, providing large datasets to be analysed; making tools available to support engagement with citizen scientists that are geographically distributed; enabling communication between citizen scientists; and providing a wider reach to a broader audience of citizen scientists.

\section{RESEARCH QUESTIONS}

While it is evident from previous research that there is a good understanding of CS and OCS from an academic perspective, what is unclear is how teachers perceive it, the methods they use to embed it in learning, and the overall impact it has on science learning for primary school children. We thus develop our three guiding research questions as follows:

RQ1. How do primary school teachers perceive OCS projects?
RQ2. Why is embedding OCS projects in primary school science learning beneficial?

RQ3. How do primary school teachers embed OCS projects in learning environments?

\section{RESEARCH METHODS}

\subsection{Multiple Case Study Design and Justification}

Our inquiry sought to answer how teachers embed web-based OCS in a classroom environment and why this improves science learning aptitudes, inquisitiveness and capabilities in primary school age children, making it very relevant to use an exploratory case study design [25]. We needed to explore and understand primary school teachers' perspectives of OCS, how they use it in classroom environments, and the overall fit it has with promoting science education whilst meeting specific project goals. By exploring a unique set of cases of four different teachers' perceptions and contextual experiences in an unfolding project, and accumulating a promisingly rich dataset, we found these elements of our study corresponding to the norms of using case study inquiry [26-29].

\subsection{Description of the Case Context, Data Sources and Methods}

To develop the perspectives that primary school teachers have of OCS, an exploratory multiple-case study design was adopted for this study. These cases involved four primary school teachers in New Zealand, who each embedded an OCS project into their teaching units. The specific learning intended in each case was determined by the teacher's particular teaching unit, which was in turn determined by a wider school plan. Once each teacher knew what unit they would be teaching during the time set for the project, they reviewed and selected a relevant OCS project(s) that could be linked with the unit topic and goals. They implemented their units over the course of a term, introducing and embedding their selected OCS project(s). Below we provide a description of the four cases (see Table 1 for a summary), an overview of the data collection activities, and conclude by explaining our data analysis methods.

Table 1: Summary of Case Studies

\begin{tabular}{|c|c|c|c|c|}
\hline Case & Teacher & Topic Focus & OCS Used & $\begin{array}{l}\text { \# of } \\
\text { Students }\end{array}$ \\
\hline Case 1 & A & $\begin{array}{l}\text { Animal } \\
\text { identificatio } \\
\mathrm{n}\end{array}$ & $\begin{array}{l}\text { Identify New } \\
\text { Zealand } \\
\text { Animals }^{4}\end{array}$ & 25 \\
\hline Case 2 & B & $\begin{array}{l}\text { Pollution in } \\
\text { streams }\end{array}$ & $\begin{array}{l}\text { The Plastic } \\
\text { Tide }^{5}\end{array}$ & 62 \\
\hline Case 3 & $\mathrm{C}$ & $\begin{array}{l}\text { Light \& } \\
\text { sound }\end{array}$ & $\begin{array}{l}\text { Agent } \\
\text { Exoplanet }^{6}\end{array}$ & 44 \\
\hline
\end{tabular}

\footnotetext{
${ }^{4}$ https://www.zooniverse.org/projects/vykanton/identify-new-zealand-animals

${ }^{5} \mathrm{https} / / /$ www.theplastictide.com/

${ }^{6}$ https://lco.global/agentexoplanet/
} 


\begin{tabular}{llll} 
& & & \& Planet \\
Hunters & & \\
Case 4 & D & $\begin{array}{l}\text { Power of } \\
\text { light }\end{array}$ & $\begin{array}{l}\text { Globe at } \\
\text { Night }^{8}\end{array}$ \\
\hline
\end{tabular}

4.2.1 Case 1 - Teacher A. The first case consisted of 25 students between the ages of 9-11, where the focus of the unit was around local animal identification, and their behavior, which ran for more than 3 weeks. Within this unit, Teacher A identified a number of learning outcomes, including: how to make observations and collect data about pests; understand how scientists collect data and make observations from it; appreciate how technology can assist in making these observations; and develop an understanding of Kaitiakitanga ${ }^{9}$ (the Māori concept of guardianship, for the sky, the sea, the land and living things.). To help achieve these outcomes, Teacher A selected an OCS project called Identify New Zealand Animals. The project aims to understand the impact that introduced mammalian predators, such as rats and stoats, have on urban environments. Citizen scientists were required to detect and classify animals on photos taken by motion-activated cameras around different New Zealand cities. The students had the added benefit of the professional scientist who setup this project coming into the classroom to discuss it with them before they started. Students initially used the project tutorial to understand what they were required to do, and then used the OCS website to classify the animals in the photos provided there. They also trapped predators at their school, an activity that Teacher A was linking with the OCS project.

4.2.2 Case 2 - Teacher B. The second case consisted of 62 students between the ages of 6-10, where the focus of the unit was on the impact of plastic in waterways (this was part of a larger project focusing on pollution in streams) and ran over twenty weeks. With this unit, Teacher B identified a number of learning outcomes, including understanding how scientists use observations to help answer questions about our world; identify and understand how humans have changed the stream habitat and water quality; identify and reflect on actions to help protect waterways. To help achieve these outcomes, Teacher B chose an OCS project called The Plastic Tide. The project aim is to build an open source machine learning algorithm that can detect plastic washed up on beaches using images captured by drones. Citizen scientists are involved in training this algorithm by classifying the plastic and litter they see in the photos provided. Teacher B initially discussed with the students where plastic comes from and the impact plastic litter has on the environment. The teacher then introduced sand samples she had collected from a local beach containing a range of plastic items. Students identified and sorted these plastics into groups of different types. Following this activity, students were introduced to the OCS project, where they were asked to tag and classify the types of litter in the photos of beaches around the world.
4.2.3 Case 3 - Teacher $C$. The third case consisted of 44 students between the ages of 11-13, where the focus of the unit was light and sound, which ran for 10 weeks. With this unit, Teacher C identified a number of learning outcomes, including: how exploration and investigation allows us to study the behaviour of light and sound; understanding of these behaviours and features; and a variety of applications of light and sound. In order to achieve these learning outcomes, the students undertook a number of activities aimed at developing their understanding of the properties of light and sound. Teacher $C$ then chose two OCS projects - Agent Exoplanet and Planet Hunters as an application of light properties. The aim of both projects is to identify exoplanets. Agent Exoplanet provides citizen scientists with images taken by telescopes to classify, while Planet Hunters provides graphs of stars' light curves to classify. Teacher C initially introduced students to exoplanet types, and used models to show how the brightness of stars can be measured by gaging the dip in light intensity that occurs when a planet passes between the star and the receiving telescope. Following these activities, students used support material provided by the teacher, Agent Exoplanet and Planet Hunters to understand the task they had to complete for each. When familiar with each, they participated in interpreting the graphs and images.

4.2.4 Case 4 - Teacher D. The fourth case consisted of 21 students between the ages of 8-10, where the focus of the unit was the power of light, and ran for 9 weeks. With this unit, Teacher D identified a number of learning outcomes, including: understanding the physics of light; how light pollution impacts the living and physical world; to get an appreciation of how science is a way of explaining the world, that science knowledge changes over time; and how to critically evaluate the reliability of data. In order to achieve these understandings, Teacher D selected an OCS project called Globe at Night. The project aims to raise public awareness of the impact of light pollution. Citizen scientists were required to collect and submit data by observing their night skies, providing information about date, time, location, and how it compared to one of 7 magnitude charts. Teacher D spent the initial 4 weeks building students' understanding of light, covering topics such as properties of light, methods for measuring light, sources of light, and light pollution. Following this, the OCS project was introduced, where Teacher D created a "treasure hunt" of the OCS website to familiarize students with the site. They then used some of the support material provided by Globe at Night, including video and documentation. While the initial plan was for students to contribute to the OCS project by taking pictures of their night skies, this was not possible due to weather conditions. However, Teacher D was able to take some pictures that they could use with the magnitude charts to categorise the degree of light pollution. Students were also asked to examine data collected over a period of years from the Globe at Night website to identify and suggest explanations for any patterns and trends, and then compare the data with images available from satellite maps, considering the reliability of each data set.

\footnotetext{
${ }^{7}$ https://www.planethunters.org/

${ }^{8} \mathrm{https} / / /$ www.globeatnight.org/
} 


\subsection{Overview of Data Collection}

Data was collected using guided interviews with teachers, their planning documentation, and collaborative workshops attended by the teachers, providing us with multiple sources of evidence from which to triangulate and validate our findings. In order to capture the teachers' experiences when embedding the OCS projects, three questionnaires were administered at three different stages: a pre-intervention stage; a mid-intervention stage; and a post-intervention stage (see Appendix B). Lastly, after completing each stage, we ran a full-day workshop with the teachers, to discuss their progress and experiences. The pre-intervention stage consisted of the teachers creating their teaching units to embed OCS, and occurred in April 2018. When this stage was completed, each of the teachers completed a questionnaire. The questions were structured to understand the teachers' view of how the OCS project they chose linked with the learning outcomes of their unit; what challenges they expect to face; and how it fits with the science capabilities of the NZ curriculum.

Teachers then implemented their teaching units, with the OCS project embedded. A mid-intervention stage in May 2018 captured the progress of embedding, with the teachers again completing an additional questionnaire. These questions were structured to understand what was working well, what difficulties they encountered, and what actions they took to support student learning with respect to the OCS project. This was followed by another workshop. Lastly, a post-intervention stage in July 2018 captured the teachers' experiences having completed the implementation of their unit, where each of the teachers completed a final questionnaire. These questions asked teachers to explain how they helped students to engage with the OCS project, what successes and challenges they experienced in using the OCS, and what information they think teachers require when considering an OCS project as part of a science unit. A final workshop was run to again discuss their experiences in greater detail. Additionally, documentation also consisted of the unit plans that each of the teachers created when planning their units, identifying how they were going to embed an OCS project in their units.

\subsection{Data Analysis}

In terms of analysis of the cases, a multi-case study approach allows for cross-case analysis and comparison, and for the phenomenon to be investigated across multiple settings [26, 29]. As the research method used was multiple-case study, cross-case synthesis is the most appropriate analytic technique that can be used for analysing the data [28]. This consisted of a thematic analysis, where an inductive coding approach was adopted, allowing themes to emerge from the data. One of the investigators coded the unit plans and questionnaires, which was then reviewed independently by two other investigators for confirmation of the emerging themes. All three agreed on the themes that had emerged from the collected data. Presented in the next section is an overview of the four cases, which is then followed by the findings and a discussion about each of them.

\section{RESULTS AND DISCUSSION}

The findings are structured according to the research questions. First we present and discuss teachers' perspectives regarding OCS projects. Here we also discuss teachers' perspectives on why embedding OCS is helpful in primary school science teaching and learning. Second, we discuss how teachers embed OCS projects and their suggestions on how embedding may be optimised. Additionally, we discuss an emergent theme of cultural inclusivity in OCS projects.

\subsection{Teachers' Perspectives on OCS Projects - Importance, Engagement \& Feedback}

Teachers expressed both interest and excitement that OCS can be a new, engaging tool and method for teaching that attracts young learners into science. Our cases revealed why embedding OCS was helpful in primary school science learning. It supported the very notion of having OCS - it attracted students towards science learning, encouraged them to approach scientific tasks and data with curiosity and supported critical thinking, inference, and learning. We had several instances during the field study when both teachers and students expressed excitement, a sense of discovery, and practical experiences that reinforced and supplemented previously learnt content.

For example, to support critical thinking and critiquing evidence capabilities, Teacher D used satellite maps along with Globe at Night to show the difference between reported light pollution from OCS and the image taken from space. This led to an important discussion on students' understanding about missing data, reliability of OCS, and reasons why light pollution for certain areas might not be reported and present on the OCS map, yet exist in real life. During the project, teachers reported that students felt drawn to being or feeling like actual "scientists".

\section{"...they are scientists and they are doing science" \\ (Case 2, Teacher B) \\ "They can go right on to interpreting the graphs, and be fully engaged and excited by the fact that they are using REAL data and that their interpretations are used by REAL scientists for a REAL purpose." \\ (Case 3, Teacher C)}

This participation can encourage the attitude of "I can do science" which is one of the pivotal points for OCS [15, p.6]. In addition, evidence from the cases showed that dissemination of results of the OCS project itself was an important feedback element. It was a critical aspect of promoting participation. While developing science capabilities and a better understanding of the scientific process are primary reasons for OCS involvement, teachers further indicated that seeing results of contributions made was also very important. They indicated the importance of being able to see the output(s) and the progress of the OCS project that the students participated in, to see how their efforts contributed to a real world scientific project, and that their active involvement had some tangible outcomes. As such, feedback regarding the output of OCS projects was expected to provide 
satisfaction for the work done, and stimulate a willingness to engage in OCS projects in the future.

"Our data will contribute to a global data set that we can then discuss and use for our own investigations. As well as being part of our Science unit, this data could be linked in with maths, literacy, social sciences, art etc." (Case 4, Teacher D)

However, this may be different from the typical goal of professional scientists. Often, professional scientists are more concerned with the positive findings in relation to their research question(s), and the publication(s) that will result from those findings. This view is especially prevalent in academic research projects in which end results are primarily in the form of scholarly publications as these are tied to rewards such as promotion and scholarly recognition. Such a view prevents some of the goals that OCS is supposed to help achieve, and possibly hinders the growth of science that can be achieved through such projects. Another issue is that the results of the project may not be easily accessible by the general public if they are presented in specialized conferences and academic journals. Scientific papers written by and for academics might also be difficult to understand for general public audiences, as the results are presented using specialist language and formatting. The reading age requirements to understand these papers is often beyond the scope of primary students.

Therefore, it is important that if professional scientists are engaging with students in their OCS projects, they need to be prepared to look beyond the academic paper. They need to engage with the students who are assisting them, disseminating the results in ways students and other non-scientists can interpret and understand. This can be achieved in numerous forms, such as presentations, student and non-scientist friendly blog posts, wikis, Q\&A sessions, and forums. Doing so can help students and others see the "bigger" picture in terms of their contributions, and helps form their opinion on science, and professional scientists themselves. For example, in the first case, Teacher A had the scientist who was running the OCS project come into the classroom and introduce it to the students, which greatly increased the interest, and engagement, in the project.

"Having the 'author' introduce the project was a real win in engagement. [He] talked about why he was doing what he was doing, the process, and how. He showed the class a motion- detecting camera as well. The class was hooked right from the start!"

(Case 1, Teacher A)

Further, by having such engagement, the citizen scientists may be more careful in terms of how they complete the task required of them, increasing accuracy of results:

"This was hugely supported I believe by having [him] come and talk about the importance of his work. The students were so excited to help him and make sure they made cautious observations - nothing rushed."

(Case 1, Teacher A)
Such engagement can also come from professional scientists who might not be part of the OCS project. For example, Teacher $\mathrm{D}$ had a professional scientist, who is an expert on the topic they were studying, to talk about it.

"Inviting [professional scientist] in to talk about light pollution and share his experience was really successful in engaging students. [He] talked about the difficulties in measuring light pollution around the globe and introduced students to the idea of using scientific measurement tools to accurately gauge light pollution levels."

(Case 4, Teacher D)

This again encourages the attitude of "I can do science" [15, p.6]. Further, it ties in with Raddick, Bracey [15] argument, which draws on Chambers [30], that students and the public become more aware of scientists, how they work, and gives them confidence that they can take on such roles in the future.

"Reminding them about the Scientist who visited and why they were doing this spurred them along. A real insight into how science can be tedious at times but persistence is important."

(Case 1, Teacher A)

"Sharing their work with scientists will add value to the work in the eyes of the children"

(Case 2, Teacher B)

Finally, students themselves can become disseminators of knowledge, where they bring their new knowledge back to the home, and community in which they are part. This is in line with the goal of curious minds [7]. It is not just about trying to create a nation of scientists, but encouraging citizens to be able to use their science knowledge to participate as critical, informed, and responsible citizens. Such endeavours can help disseminate scientific knowledge in a local context.

"We shared what we were doing with parents via class blog and class newsletter. Lots of interest!"

(Case 1, Teacher A)

"It gave the children a global appreciation of a local problem and has encouraged them to pursue their local study and present their findings to our local community." (Case 2, Teacher B)

\subsection{Embedding OCS in Learning Environments \& Suggestions for Improvement}

When setting up an OCS project, there are many aspects that need to be considered such as: choosing the research question(s) to be addressed; forming a project team (if necessary); and developing, testing, and refining protocols to be followed by the citizen scientists [21]. While OCS projects outside school environments have found that these aspects may also involve participating citizen scientists, we found that in a primary school environment, teachers and their classes are not necessarily interested in these 'project' aspects. Instead, it was much easier for the professional scientist(s) to set up these aspects, such as creating relevant research questions based on their experience and knowledge of 
what they want to study. In addition to their normal duties, attempting to include teachers in OCS project planning appeared to stretch their already limited time. We found that teachers are interested in the material that helps them and their classes to understand (i) the requirements of a project, (ii) if it is suitable for their students, and (iii) how it can fit in their classrooms. This was evidenced by the teachers already considering this in the preimplementation stage, when they were looking at OCS projects that would be a good fit for their scenarios. For example, in the first case, Teacher A was considering the tutorials that their potential OCS project provided for new participants when reviewing suitable projects. More so, all the four teachers in our cases indicated that they found explanatory material provided to be very helpful in their strategies for embedding the projects into student learning activities.

"The tutorial provided by the OCS project was also very helpful."

(Case 1, Teacher A)

"Introduce The Plastic tide: Share how to use the website." (Case 2, Teacher B)

However, an issue that arose in the fourth case, was that while the OCS project provided support in the form of videos and documentation, Teacher D's class found the material to be difficult to understand. Although the documentation was provided for students to help understand the work that was required of them, issues arose with students not being able to read or understand it, particularly with regards to the technology being used. This may be not only because they had limited understanding of science in general, but also that the supporting material may have been pitched higher than primary school student level.

Therefore, it is important for professional scientists to carefully and properly prepare supporting material. Such material should be simple enough to convey the intended message that the targeted recipients can understand. Training materials that are richer in content, including images, videos, and other interactive content, that complement each other were reported to provide a better explanation than plain text only. Additionally, teachers indicated that interactive user interfaces (UI) and engaging user experiences (UX) provided by the design of the web-based platforms through which students access and use material in an OCS project can have a positive effect on students' initial impression, willingness to actively participate, and overall engagement throughout a project. For instance, Teacher A reported that,

"I was thrilled with how everyone was engaged right from the start! Able students and those less able. This was a wonderful UDL ${ }^{10}$ tool - the online hook -in and clear instructions with check boxes that meant everyone could understand what to do."

(Case 1, Teacher A)
Therefore, in creating supporting material, professional scientists should consider their audience, richness of the material, UI \& UX design, and the appropriate level of detail required. By making such information explicit, and providing engaging tools, teachers would better understand the project as a whole, and successfully embed it in teaching and learning activities in their classroom environments. Such practice may also encourage greater participation from general citizens.

We further asked teachers to consider what information they looked for from an OCS project in order for them to select an appropriate one for use in their classroom. Suggestions that were provided can be seen in Table 2. The most common suggestions included the timeframe of when students could contribute to the project, the age range of a project, technology requirements of a project, and the science skills that are supported in participating in the project. These were the critical elements that teachers needed to successfully embed OCS in primary school learning environments.

Table 2: Suggestions from teachers about information required about OCS projects

\begin{tabular}{|c|c|}
\hline Case & Suggestions from Teachers \\
\hline Case 1 , Teacher A & $\begin{array}{l}\text { - } \text { Length of time for project } \\
\text { - } \\
\text { - } \\
\text { - } \quad \text { Curriculum links } \\
\text { Ways to integrate with other } \\
\text { - Tearning areas } \\
\text { Technology requirements }\end{array}$ \\
\hline Case 2, Teacher B & $\begin{array}{l}\text { - Contexts that the project could be } \\
\text { meaningfully linked to } \\
\text { Estimated age ranges the project is } \\
\text { appropriate for } \\
\text { - Science skills or capabilities that } \\
\text { are supported by the use of the } \\
\text { project } \\
\text { - What links can be made to local / } \\
\text { hands on activities } \\
\text { The science strands that are } \\
\text { covered by the project }\end{array}$ \\
\hline Case 3, Teacher C & $\begin{array}{ll}\text { - } & \text { Curriculum strand } \\
\text { - } & \text { Nature of Science strands } \\
\text { - } & \text { Science Capabilities developed } \\
\text { - } & \text { Broad age range / curriculum level } \\
\text { - } & \text { Ideas / stories / resources from } \\
& \text { teacher who have used it } \\
\text { - } & \text { Technology requirements }\end{array}$ \\
\hline
\end{tabular}

${ }^{10}$ Universal Design for Learning - designing learning experiences inclusive of students with special learning needs 


\begin{tabular}{ll}
\hline Case 4, Teacher D & A general idea of the age and \\
curriculum range the project is \\
most appropriate for \\
An approximate reading age for \\
the material supplied with the \\
project \\
The type of engagement supported \\
(data gathering, interpretation etc.) \\
and the timeframe and level of \\
commitment required \\
Can individuals access their own \\
data? Are contributions or progress \\
tracked? Can the data be taken \\
from the project to be used in \\
different contexts?
\end{tabular}

Considering the feedback from our four primary school teachers, we developed a set of criteria that professional scientists should consider if they want to attract teachers to use their OCS projects in their classrooms. We then had cycles of iterative feedback meetings with the teachers, discussing the criteria, to confirm and refine them. This was intended to ensure that each criterion represents the teachers' needs and expectations from OCS projects. These criteria are presented and explained in Table 3. Having explicit information about each criterion allows teachers to better understand the OCS project, its potential relevance to their students, and how to successfully embed it in teaching and learning. As OCS proliferates in primary level learning environments, the criteria are an important finding for productive engagement between professional scientist(s) (who are clients) and the school teachers and their classes (who are the hosts).

Of course, a question that might be posed is why scientists should care about their OCS projects being used by primary school children? We argue that school children are the future, and embedding OCS projects in their learning process, inculcates aptitudes for science learning that may encourage them to get involved with science and even pursuing science careers in the future. Additionally, the more participants for a project, the more beneficial it is for the scientists, and the OCS platform. Using OCS in a school is one way of encouraging this participation with a simultaneous benefit to the learners and higher participation and contributions for OCS projects.

\subsection{Cultural Inclusivity in OCS}

Our study was carried out in New Zealand. While there is a significant overlap between the New Zealand curriculum to the curricula of other countries, New Zealand features a unique bicultural context that recognizes the special place of indigenous Māori culture. Hence, cultural responsiveness of the online citizen science projects that are to be selected for learning activities is a major concern to teachers and students alike. At the time of this study, we were not aware of OCS projects that incorporate any form of cultural responsiveness. We thus found this to be
Table 3: Important contextual descriptions required for an OCS project to be used in the classroom

\begin{tabular}{|c|c|}
\hline Context & Explanation \\
\hline $\begin{array}{l}\text { Purpose of } \\
\text { the project }\end{array}$ & $\begin{array}{l}\text { Provide a general overview of the project, } \\
\text { explaining the purpose, and what it wants to } \\
\text { achieve, including the area of focus }\end{array}$ \\
\hline $\begin{array}{l}\text { Project } \\
\text { timeframe }\end{array}$ & $\begin{array}{l}\text { State when the project will start, how long it } \\
\text { will run for, what stages participants will be } \\
\text { required to complete their tasks (including } \\
\text { when they will be expected to do so), and } \\
\text { when and how results will be disseminated }\end{array}$ \\
\hline $\begin{array}{l}\text { Requirement } \\
\mathrm{s}\end{array}$ & $\begin{array}{l}\text { Technical requirements such as: } \\
\text { - } \quad \text { Devices that can be used } \\
\text { - } \quad \text { App(s) and software needed } \\
\text { - } \quad \text { Accessibility (is an individual account } \\
\text { Skequired, or will a group one do?) } \\
\text { - } \quad \text { Numeracy skills } \\
\text { - } \quad \text { Literacy skills } \\
\text { - } \quad \text { Technical/Computer skills }\end{array}$ \\
\hline $\begin{array}{l}\text { Support } \\
\text { provided }\end{array}$ & $\begin{array}{l}\text { Specify what support will be provided for the } \\
\text { participants for the different stages of the } \\
\text { project. This includes materials that are } \\
\text { available to gain an understanding of the } \\
\text { project, what's expected of the participants, } \\
\text { such as videos and/or documentation }\end{array}$ \\
\hline Age level & $\begin{array}{l}\text { Indicate what the minimum suitable age level } \\
\text { for participation is }\end{array}$ \\
\hline $\begin{array}{l}\text { Relevance to } \\
\text { students' } \\
\text { learning }\end{array}$ & $\begin{array}{l}\text { Explain the area of focus for the project, what } \\
\text { aspects of this area will be touched on during } \\
\text { the project, and how students will be } \\
\text { exposed to it by their participation }\end{array}$ \\
\hline $\begin{array}{l}\text { Geographic } \\
\text { scope }\end{array}$ & $\begin{array}{l}\text { Indicate if the OCS project is of local, national, } \\
\text { or international interest }\end{array}$ \\
\hline $\begin{array}{l}\text { Data } \\
\text { availability }\end{array}$ & $\begin{array}{l}\text { Indicate if the data that is gathered, or used, } \\
\text { in the project is available for access outside of } \\
\text { the project itself for further manipulation, } \\
\text { such as being able to collaboratively compare } \\
\text { it with other classes/schools locally, } \\
\text { nationally, and/or internationally }\end{array}$ \\
\hline
\end{tabular}

revelatory. This could start from being available in other languages than just English (which does already exist in some projects), but could go as far as a careful consideration of what may be regarded as sacred topics in indigenous cultures, or even to questioning the nature of the scientific inquiry and the adequacy of the underlying knowledge system [31]. For example, all the teachers described culturally responsive practices in embedding OCS: 
"We talked about our school RIMU11 values - especially Integrity and how as citizen scientists we had to show integrity with our observations."

(Case 1, Teacher A)

"Whole-school focus on Matariki12 - connect by talking about how light travels through space. Polynesian peoples navigated by the light of the stars and used the light of stars as their calendar. Today we know about the universe through observing and measuring light from stars." (Case 3, Teacher C)

"Link with Matariki - students will identify and observe the Matariki constellation and explore how astronomical observations were used to measure time."

(Case 4, Teacher D)

This is further explained by Teacher B, who introduced a type of learning that encourages a traditional Māori model of learning:

"Use a tuakana / teina 13 model of learning so older children are supporting the younger children"

(Case 2, Teacher B)

While this seems to be a major cultural shortcoming of existing OCS projects, it can equally be a great opportunity for a largescale participatory exercise. Teachers can pick up the topics represented by the different OCS projects and ask their students to talk to senior citizens of their indigenous communities to provide their stories about these topics, e.g. in New Zealand the Matariki and navigation stories about the stars at night.

"I used the context of constellations as an opportunity to explore traditional stories. Before we began to explore the OCS students were asked to find traditional stories from their own cultures relating to the stars or constellations for homework. Despite a very culturally diverse class, many students struggled to find stories from their own cultures and instead chose stories relating to the Greek and Zodiac constellations familiar in Western culture. However, one student shared a Māori story (a legend of Matariki) and other shared Sri Lankan, Samoan and Chinese legends"

(Case 4, Teacher D)

This would not only be a genuine enrichment of the citizen science landscape but would contribute to the importance of acknowledging this knowledge as being owned by the indigenous people of an area. This further contributes to valuing citizens for their many knowledge systems and validating their place in citizen science.

\section{CONCLUSION}

As the trend towards the use of OCS projects in education is increasing, with a promising potential to provide rich experiences in science teaching and enhancing science learning aptitudes and capabilities in particular, it is important to have a closer look at how the participants of such projects view and are affected by

\footnotetext{
11 Related to Māori values and native forest

${ }^{12}$ Māori name for the cluster of stars also known as the Pleiades

http://tereomaori.tki.org.nz/Curriculum-guidelines/Teaching-and-learning-tereo-Maori/Aspects-of-planning/The-concept-of-a-tuakana-teina-relationship
}

them. Our exploratory study is the 'first of its kind' in doing so. It provides early insights that provoke reflection about the perspectives of primary school teachers regarding OCS. We find that at primary school level, OCS provides unique opportunities for teachers and their students to engage with professional scientists in learning environments, to stimulate interest and curiosities about science in young learners, and to support the learning and acquiring of critical skills and capabilities. It provides the criteria that are important for productive and successful engagement between professional scientist(s), school teachers and their classes. The study further provides some initial insights into the importance of rich content as well as the use of engaging UI and interactive UX designs in web-based OCS platforms intended for a primary school classroom environment. Additionally, OCS projects in educational environments should have feedback cycles in which learners are updated about the impact of their previous engagements. This provides young learners with a sense of real scientific contribution which goes beyond the immediate learning activities and reinforces aptitudes towards science learning. Therefore, our findings and associated recommendations provide professional scientists with insights about how to engage school teachers and the meaningful ways in which such engagements can be productive and successful to both science learning in schools and OCS-enabled scientific research projects. These findings are a significant step towards an understanding of OCS in teaching and learning at the primary school level.

There are some limitations to the study. First is that the focus is on the NZ curriculum, which has its own peculiarities that may not be applicable to other countries. We also acknowledge the limitation of the scope with the four cases in this exploratory study, considering that the findings are based on the teachers' perspectives only. However, as this is part of a bigger research project, we have ongoing endeavours to expand this scope. In particular, we will include the student's perspectives and intend to expand our assessment and evaluation of the benefits of OCS in teaching and learning. Overall, the provoking insights emerging from this exploratory study are difficult to ignore and can be a start in building blocks towards successful OCS projects from both the perspectives of professional scientists and teachers and learners in educational environments.

\section{ACKNOWLEDGEMENTS}

This work is funded by Teaching and Learning Research Initiative (TLRI) of New Zealand under Project No. 9182. ${ }^{14}$ TLRI supports and enhances links between educational research and teaching practices to improve outcomes for learners.

The authors would also like to thank the anonymous reviewers for their valuable comments and helpful suggestions.

\footnotetext{
14 http://www.tlri.org.nz/tlri-research/research-progress/school-sector/citizenscientists-classroom-investigating-role-online
} 


\section{REFERENCES}

[1] Tinati, R., M. Van Kleek, E. Simperl, M. Luczak-Rösch, R. Simpson, and N. Shadbolt. 2015. Designing for citizen data analysis: A cross-sectional case study of a multi-domain citizen science platform. In Proceedings of the 33rd Annual ACM Conference on Human Factors in Computing Systems (CHI '15). ACM Press, 4069-4078.

[2] Tinati, R., M. Luczak-Roesch, E. Simperl, and W. Hall. 2017. An investigation of player motivations in Eyewire, a gamified citizen science project. Computers in Human Behavior, 73, 527-540.

[3] Jay, C., R. Dunne, D. Gelsthorpe, and M. Vigo. 2016. To sign up, or not to sign up?: maximizing citizen science contribution rates through optional registration. In Proceedings of the 2016 CHI Conference on Human Factors in Computing Systems. ACM, 1827-1832.

[4] Tinati, R., M. Luczak-Roesch, E. Simperl, and W. Hall. 2016. Because science is awesome: studying participation in a citizen science game. In Proceedings of the 8th ACM Conference on Web Science. ACM, 45-54.

[5] Oliveira, N., E. Jun, and K. Reinecke. 2017. Citizen Science Opportunities in Volunteer-Based Online Experiments. In Proceedings of the $2017 \mathrm{CHI}$ Conference on Human Factors in Computing Systems. ACM, 6800-6812.

[6] Law, E., K.Z. Gajos, A. Wiggins, M.L. Gray, and A.C. Williams. 2017 Crowdsourcing as a Tool for Research: Implications of Uncertainty. In CSCW. 1544-1561.

[7] What is Curious Minds? Available at: https://www.curiousminds.nz/about/ Last access: 15th February 2019.

[8] 2007. Ministry of Education. The New Zealand curriculum. Wellington: Learning Media.

[9] Hofstein, A. and V.N. Lunetta. 1982. The role of the laboratory in science teaching: Neglected aspects of research. Review of educational research, 52(2), 201-217.

[10] Hofstein, A. and V.N. Lunetta. 2004. The laboratory in science education Foundations for the twenty-first century. Science education, 88(1), 28-54.

[11] Bolstad, R. and R. Hipkins. 2008. Seeing yourself in science. Wellington, New Zealand: New Zealand Council For Educational Research.

[12] Tytler, R., D. Symington, V. Kirkwood, and C. Malcolm. 2008. Engaging students in authentic science through school--community links: learning from the rura experience. Teaching Science: The Journal of the Australian Science Teachers Association, 54(3)

[13] Caygill, R., V. Hanlar, and C. Harris-Miller. 2016. Student attitudes to maths and science: what we know from New Zealand's TIMSS 2014/15 results for Year 5 and Year 9. Retrieved 15th February, 2019 from: http://www.educationcounts.govt.nz/publications/series/2571/timss-201415/student-attitudes-tomaths-and-science-what-we-know-from-new-zealands-timss-201415-resultsfor-year-5-and-year-9.

[14] Educational Assessment Research Unit and New Zealand Council for Educational Research. Wānangatia te Putanga Tauira National Monitoring Study of Student Achievement Science. 2012. Available at: http://nmssa.otago.ac.nz/-reports/2012_Science_ONLINE.pdf. Last access: 15th February 2019.

[15] Raddick, M.J., G. Bracey, K. Carney, G. Gyuk, K. Borne, J. Wallin, S. Jacoby, and A. Planetarium. 2009. Citizen science: status and research directions for the coming decade. AGB Stars and Related Phenomenastro 2010: The Astronomy and Astrophysics Decadal Survey, 2010, 46P

[16] Raddick, M.J., G. Bracey, P.L. Gay, C.J Lintott, C. Cardamone, P. Murray, K. Schawinski, A.S. Szalay, and J. Vandenberg. 2013. Galaxy Zoo: Motivations of citizen scientists. arXiv preprint arXiv:1303.6886.

[17] Crowston, K., E. Mitchell, and C. Østerlund. 2018. Coordinating Advanced Crowd Work: Extending Citizen Science. In Proceedings of the 51st Hawaii International Conference on System Sciences.

[18] Wiggins, A. and K. Crowston. 2011. From conservation to crowdsourcing: A typology of citizen science. In Proceedings of the 44th Hawaii International Conference on System Sciences. IEEE, 1-10.

[19] Hassman, K., G. Mugar, C. Østerlund, and C. Jackson. 2013. Learning at the seafloor, looking at the sky: The relationship between individual tasks and collaborative engagement in two citizen science projects. In proceedings for 10th International Conference on Computer Supported Collaborative Learning.

[20] Nov, O., O. Arazy, and D. Anderson. 2011. Dusting for science: motivation and participation of digital citizen science volunteers. In Proceedings of the 2011 iConference. ACM, 68-74

[21] Lewenstein, B.V. 2004. What does citizen science accomplish.

[22] Yadav, P. and J. Darlington. 2017. Conceptual Frameworks for Building Online Citizen Science Projects. arXiv preprint arXiv:1704.05084.

[23] Curtis, V. 2014. Online citizen science games: opportunities for the biological sciences. Applied \& translational genomics, 3(4), 90-94

[24] Raddick, M., G. Bracey, P. Gay, C. Lintott, P. Murray, K. Schawinski, A. Szalay, and J. Vandenberg. 2010. Galaxy zoo: Exploring the motivations of citizen science volunteers. Sept. Astronomy Education Review, 9(1).

[25] Yin, R.K. 2009. Case study research: Design and methods (applied social research methods). London and Singapore: Sage.
[26] Benbasat, I., D.K. Goldstein, and M. Mead. 1987. The case research strategy in studies of information systems. MIS quarterly, 11(3), 369-386.

[27] Eisenhardt, K.M. 1989. Building theories from case study research. Academy of management review, 14(4), 532-550.

[28] Yin, R.K. 1994. Case study research: design and methods. 1994. Thousand Oaks, CA.

[29] Darke, P., G. Shanks, and M. Broadbent. 1998. Successfully completing case study research: combining rigour, relevance and pragmatism. Information systems journal, 8(4), 273-289.

[30] Chambers, D.W. 1983. Stereotypic images of the scientist: The Draw-a-Scientist Test. Science education, 67(2), 255-265.

[31] Smith, L.T., Decolonizing methodologies: Research and indigenous peoples. 2013: Zed Books Ltd. 